\title{
Diva strategies for avian influenza
}

\begin{abstract}
In concern for animal diseases, the goal of vaccination is to prevent or reduce clinical diseases associated with the infectious agent, but it can also be used as a means of managing or eradicating a disease from a particular region. In outbreak situations, it is therefore important to be able to detect active infection in vaccinated animals. It was to satisfy this requirement that the term Differentiation of Infected from Vaccinated Animals (DIVA) was coined in 1999 by Jan T van Oirschot. ${ }^{1}$ This term is now used replacing the older 'marker vaccines'. The DIVA principle has now also been extended to include subunit and killed whole virus vaccines. ${ }^{2}$ DIVA principle is based on a DIVA vaccine producing an antibody response that is different from the antibody response produced by the wild-type virus. More recently, the use of genetic DIVA has been introduced, which is based on identifying genetic differences between live vaccines and the field viruses.
\end{abstract}

Keywords: avian, birds, antigens, virus, polymerases
Volume 3 Issue I - 2018

\author{
Rabyia Javed \\ Guru Angad Dev Veterinary and Animal Sciences University, \\ India \\ Correspondence: Rabyia Javed, Ph D Scholar, Guru Angad \\ Dev Veterinary and Animal Sciences University (GADVASU), \\ Ludhiana, Punjab, India, Email waquar1975@rediffmail.com
}

Received: January 03, 2018 | Published: January 22, 2018
Abbreviations: DIVA, differentiation of infected from vaccinated animals; AIVs, avian influenza viruses; NEP, nuclear export protein; HPAI, highly pathogenic AI

\section{Diva in avian influenza}

Avian influenza viruses (AIVs) are single-stranded RNA viruses belonging to the Influenza virus $A$ genus of the Orthomyxoviridae family. The segmented genome encodes ten or 11 proteins, depending on the strain. These proteins are the prominent outer surface glycoproteins HA and neuraminidase (NA), the matrix (M2) ion channels, the matrix (M1) protein, which is found lining the inner side of the lipid envelope of the virus, three polymerases (PA,PB1 and PB2), one NSP (NS1), a nuclear export protein (NEP) and a recently described accessory protein (PB1-F2) with pro-apoptotic activity. ${ }^{3}$ AIVs can be classified into different subtypes based on the possession of one of the 16 antigenically distinct HA antigens, typed from $\mathrm{H} 1$ to H16, and one of nine NA antigens, typed from N1 to N9. Clinically, AIVs can be classified as low-pathogenic AI (LPAI), and highly pathogenic AI (HPAI) on the basis of the severity of the disease they cause in susceptible birds.

In addition to direct control measures based on biosecurity, restriction policies and stamping out the disease, the appropriate use of vaccines is encouraged to maximize eradication efforts. It is known that vaccination prevents clinical disease, increases resistance to infection and reduces virus shedding levels, but does not prevent infection if birds are challenged with a sufficiently high dose of virus. ${ }^{4,5}$ For this reason, vaccinated birds may still become infected and shed virus into the environment without displaying any clinical signs and therefore represent a means of spreading infection. In order to achieve the goal of eradication, DIVA vaccination strategies have been recommended and must be implemented. These systems, coupled with an appropriate monitoring system, enable the detection of field exposure in vaccinated flocks, and through this, infected flocks may be properly managed. There are a number of different DIVA systems currently available or in the process of development and validation. One type is Heterologous NA-type DIVA. It is a DIVA strategy based on the use of an inactivated oil-emulsion vaccine containing the same HA subtype as the challenge virus, but a different NA. ${ }^{6}$ Another one is Nonstructural 1 protein DIVA, a promising system based on the detection of antibodies against a specific antigen, the NS1 protein of AI, has been deemed a good DIVA candidate. The NS1 protein is synthesized in large amounts in infected cells but is not incorporated into the mature virions, and for this reason represents the ideal candidate to elicit a specific immune response.

\section{Conclusion}

Probably the simplest DIVA system at a technical level is the use of sentinel birds to distinguish vaccinated and uninfected from vaccinated and infected birds. The use of unvaccinated birds in the shed has been used to identify field exposure within a vaccinated population, regardless of the strain used in the vaccine. This system requires the identification of unvaccinated birds and regular clinical inspections, together with serological testing. The approach is considered to be valid, although it requires significant preparatory work and is time-consuming, especially when the number of flocks to be vaccinated is high and when sentinel birds are not confined to cages within the flock.

\section{Acknowledgements}

The author declares there are no acknowledgments.

\section{Conflict of interest}

The author declares no conflict of interest.

\section{References}

1. Van Oirschot JT. Diva vaccines that reduce virus transmission. $J$ Biotechnol. 1999;73(2-3):195-205.

2. Pasick J. Application of DIVA vaccines and their companion diagnostic tests to foreign animal disease eradication. Anim Health Res Rev. 2004;5(2):257-262.

3. Bouvier NM, Palese P. The biology of influenza viruses. Vaccine. 2008;26(Suppl 4):D49-D53.

4. Swayne DE, Suarez DL. Highly pathogenic avian influenza. Rev Sci Tech. 2000;19(2):463-482. 
5. Capua I, Terregino C, Cattoli G, et al. Increased resistance of vaccinated turkeys to experimental infection with an $\mathrm{H} 7 \mathrm{~N}$ low-pathogenicity avian influenza virus. Avian Pathol. 2004;33(2):158-163.
6. Capua I, Terregino C, Cattoli G, et al. Development of a DIVA (Differentiating Infected from Vaccinated Animals) strategy using a vaccine containing a heterologous neuraminidase for the control of avian influenza. Avian Pathol. 2003;32(1):47-55. 